\title{
AVALIAÇÃO AMBIENTAL DE UM CICLO DE DESTILAÇÃO DE ETANOL ATRÁVES DE CÁLCULO DE EXERGIAS DE CADA COMPONENTE
}

Rafael Henrique Pinto e Silva - rhpsrafaelhenrique@ gmail.com Pontifícia Universidade Católica de Minas Gerais 


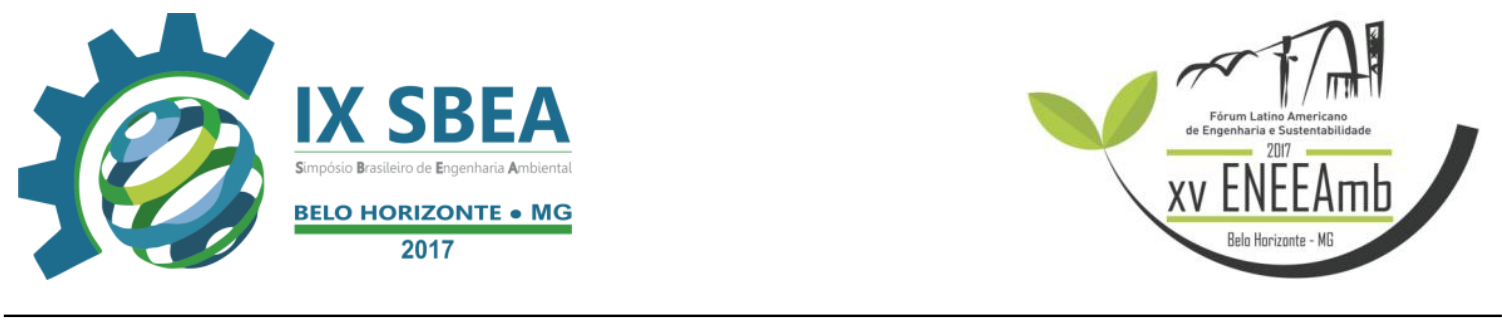

\section{RESUMO}

Este documento apresenta informações referentes a avaliação ambiental de um ciclo de destilação de etanol. O ciclo foi redesenhado a partir do programa COCO, e desenvolvido na Northern Arizona University, situada em Flagstaff. O autor é responsável pelo uso do programa para cálculo das exergias de cada componente do ciclo.

Palavras-chave: COCO, Exergia, Northern Arizona University.

\section{INTRODUÇÃO/OBJETIVO}

O conceito de exergia é a capacidade de produzir trabalho. Este conceito é ligado a irreversibilidade dos processos, a transformação da energia em trabalho, e a indicação do limite até onde a energia pode ser útil.

Devido ao crescimento de estudos baseados na relação de energia e meio ambiente, vários especialistas (pesquisadores, cientistas) sugeriram o conceito de exergia para analisar os impactos do meio ambiente graças a utilização dos recursos energéticos (Pires, 2007).

O objetivo deste trabalho é avaliar o grau de impacto ambiental de uma síntese de metanol, através dos cálculos de exergias. A síntese de metanol foi simulada na Northern Arizona University, através do software COCO. Este trabalho é um extensão do uso do COCO, com uma finalidade ambiental.

\section{METODOLOGIA}

Para o estudo, foi utilizado como base um estudo feito por professores do departamento de Engenharia Mecânica da Northern Arizona University. Este estudo consiste na simulação de uma síntese de metanol, cujo sistema foi ilustrado utilizando-se o software COCO.

A figura 1 a seguir ilustra a unidade de síntese de metanol, cujos dados foram extraídos para a ilustração no software COCO. Os números na figura representam cada componentes, sendo que alguns não foram representados tanto no arquivo feito pelo Dr. Morgan, quanto pelo autor do artigo: 1 é onde ocorrerá a eletrolise, 2 é aonde está armazenados os gases de síntese, 3 é o regulador de baixa pressão, 4 é o compressor, 5 é 


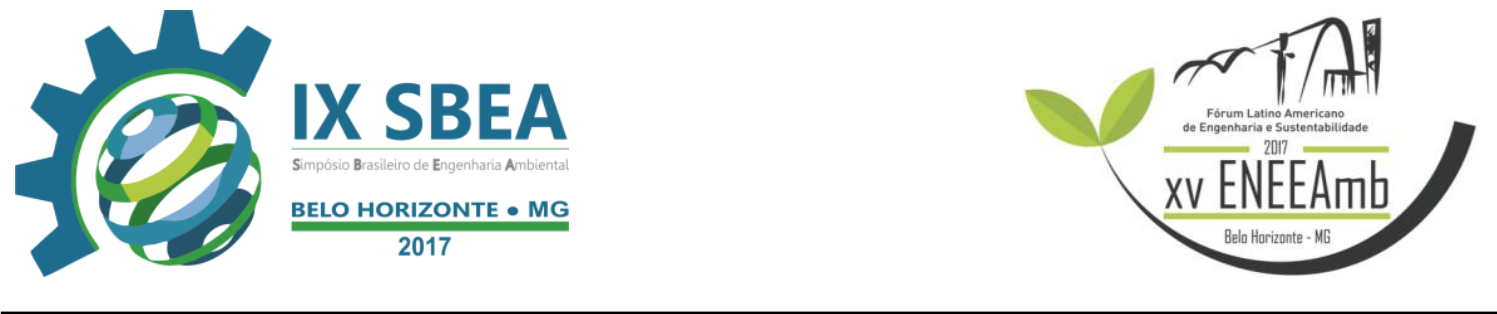

o compressor de alta pressão, 6 é o reator de síntese, 7 é a válvula de expansão, 8 é o condensador, 9 é o separador de vapor, 10 é a coluna de destilação, e 11 é a caixa de fusíveis.

Figura 1- Mecanismo de síntese do metanol

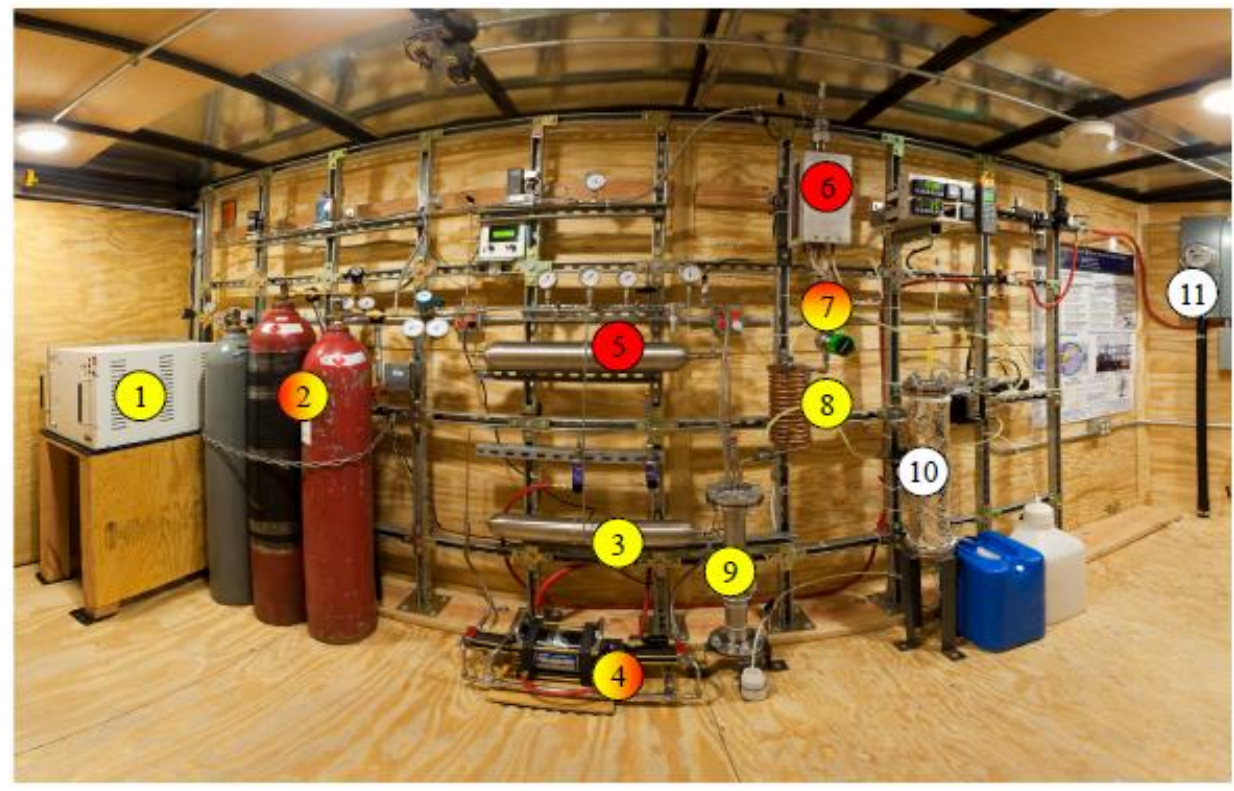

Fonte: Practical Experience with a Mobile Methanol Synthesis Device

Com o mesmo software, o processo foi refeito, mas com medidores cujos dados foram utilizados para a obtenção dos valores tanto das energias, quanto das exergias de cada componente. A figura 2 demonstra o sistema antes de ser refeito pelo autor do trabalho, para os cálculos tanto da energia quanto da exergia. 
Figura 2 - Desenho original da síntese de Metanol com o software COCO

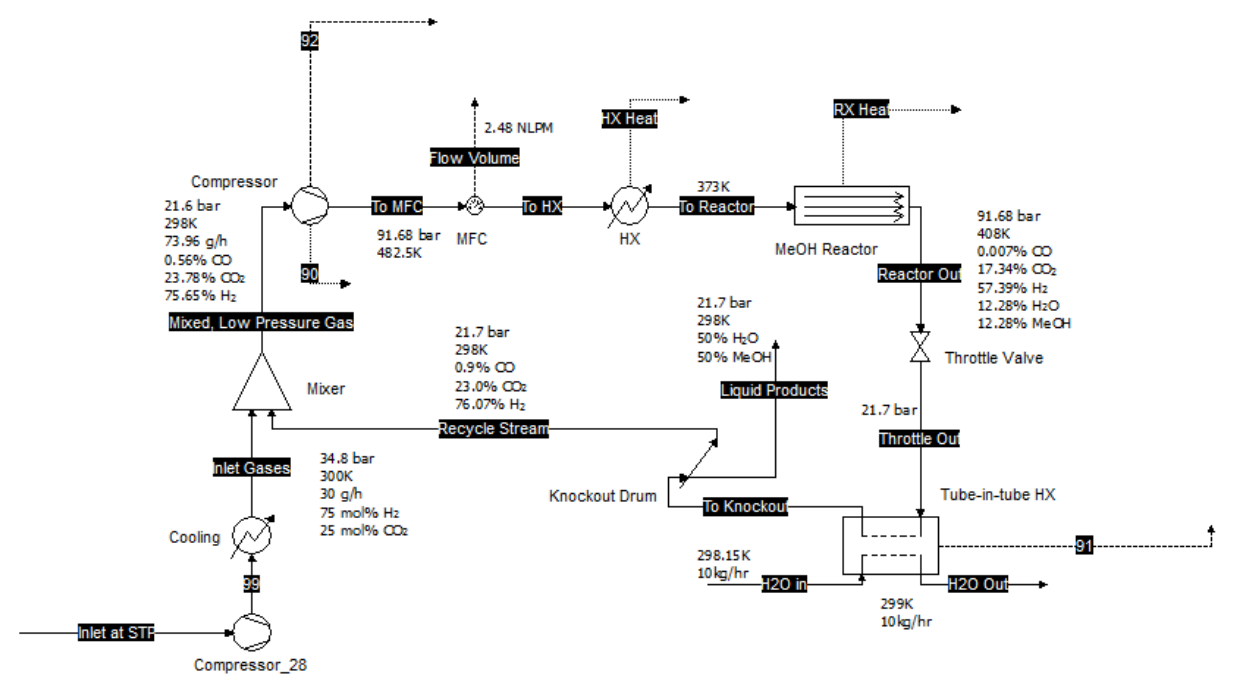

Fonte: Practical Experience with a Mobile Methanol Synthesis Device

Os cálculos utilizando-se o software foram baseados na primeira e segunda lei da Termodinâmica. Em cada componente, foi introduzida uma equação relacionada a quantidade de exergia destruída ou o fluxo de exergia do ciclo. Pela primeira lei, tem-se a expressão:

$$
E_{2}-E_{1}=Q-W
$$

Em que E2 e E1 são as energias de saída e entrada, respectivamente, e Q é o calor gerado e W o trabalho realizado. Todas esses valores estão em W. No geral, a exergia em um ponto é definida por:

$$
E=m \times(h-T \times s)
$$

Em que m é o fluxo de massa em $\mathrm{kg} / \mathrm{s}$, s a entropia correspondente em $((\mathrm{J} /(\mathrm{kg} \times \mathrm{K}))$, e T a temperatura ambiente (em K). Para o cálculo do fluxo de energia em um componente em que há trabalho, é utilizada a seguinte expressão no COCO:

$$
E x=W-E_{2}-E_{1}
$$

Já nos componentes onde existe trocas de calor, a exergia correspondente será calculada pela seguinte expressão: 


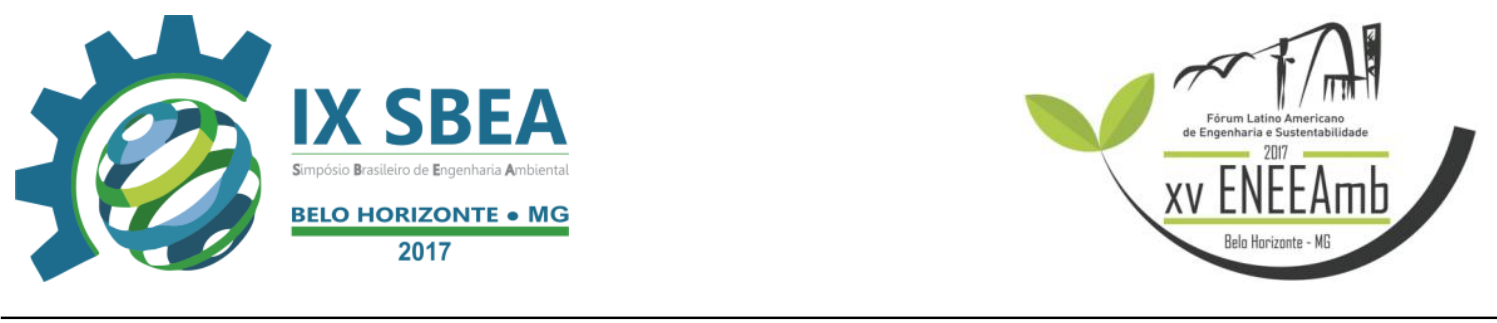

$$
E x=E_{2}-E_{1}+\left(1-\frac{T_{A}}{T_{B}}\right) \times Q
$$

Em que TA e TB correspondem, respectivamente, as temperaturas de entrada e saída, em K. Finalmente, para o cálculo do fluxo de exergia do reator de síntese do metanol, foi calculado primeiramente a exergia de cada uma das reações computadas no reator do programa.

$$
\begin{gathered}
\mathrm{CO}_{2}+3 \mathrm{H}_{2} \rightarrow \mathrm{MeOH}+\mathrm{H}_{2} \mathrm{O} \\
\mathrm{CO}_{2}+\mathrm{H}_{2} \rightarrow \mathrm{CO}+\mathrm{H}_{2} \mathrm{O}
\end{gathered}
$$

Infelizmente o software não apresenta os valores de exergia de cada componente, logo foi necessário uma consulta externa para o obtenção destes valores e grava-los no software a seguir para os posteriores cálculos. A tabela 1 mostra as seguintes exergias químicas de cada componente, utilizadas para o cálculo da exergia da reação dos produtos (MeOH e CO).

Tabela 1 - Exergia química padrão de cada substâncias das reações

\begin{tabular}{|c|c|}
\hline Gás & Exergia química $\mathbf{( k J / m o l )}$ \\
\hline CO2 & 19,48 \\
\hline H2 & 236,09 \\
\hline H2O & 0,9 \\
\hline CO & 274.71 \\
\hline MeOH & 720 \\
\hline
\end{tabular}

Fonte: SHAPIRO (2004)

Para o cálculo da exergia de formação produtos, foi igualado o somatório da exergia dos produtos mais o somatório da exergia dos reagentes.

$$
\begin{gathered}
\mathrm{M} \times\left(\mathrm{CO}_{2}+3 \mathrm{H}_{2}-\mathrm{MeOH}-\mathrm{H}_{2} \mathrm{O}+\mathrm{R} \times \mathrm{T} \times \log \frac{m f \mathrm{H}_{2}{ }^{3}}{\mathrm{mfMeOH} \times m \mathrm{H}_{2} \mathrm{O}}\right) \rightarrow \text { Exergia de } \\
\mathrm{MeOH}
\end{gathered}
$$

$$
\mathrm{M} \times\left(\mathrm{CO}_{2}+\mathrm{H}_{2}-\mathrm{CO}-\mathrm{H}_{2} \mathrm{O}+\mathrm{R} \times \mathrm{T} \times \log \frac{m f \mathrm{H}_{2}}{m f C O \times m f \mathrm{H}_{2} \mathrm{O}}\right) \rightarrow \text { Exergia de } \mathrm{CO}
$$

Em que M é a massa molar da mistura (em $\mathrm{kg} / \mathrm{kmol})$, mf é a fração molar de cada substância, R é a constante dos gases $(9,314598 \mathrm{~kJ} / / \mathrm{K} \times \mathrm{kmol})$, e T a temperatura ambiente em K. 


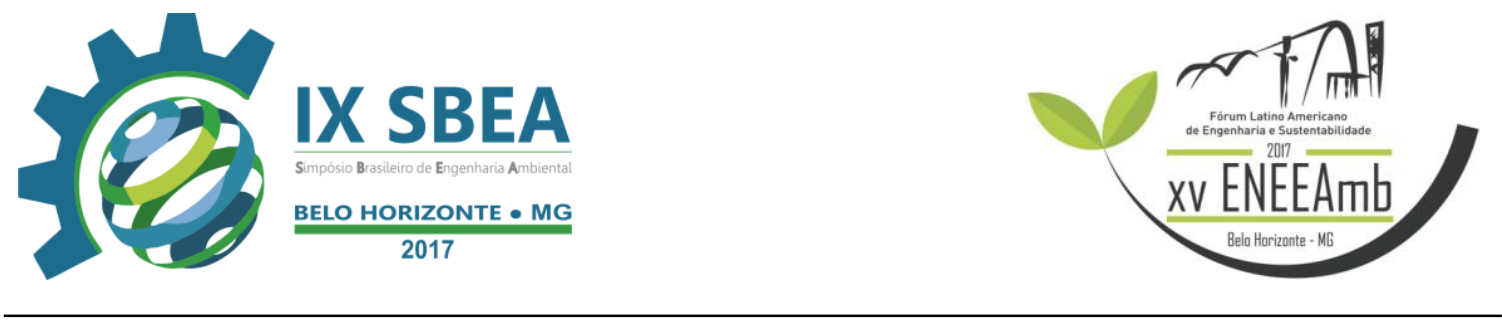

Após os cálculos de exergia de formação dos produtos, é calculado a exergia na entrada e na saída igual os processos anteriores (exergia física), mas como na reação a porcentagem de cada componente altera, um novo cálculo é computado para saber o novo fluxo de massa. Enfim, a expressão para o fluxo de exergia do reator fica da seguinte forma:

$$
E x=E_{1}-E_{2}-\left(E_{a}-E_{b}\right)
$$

Em que Ea e Eb são as exergias químicas dos produtos.

Após o cálculo do fluxo de energia e exergia de todos os componentes, os valores foram tabelados utilizando-se uma planilha do Microsoft Excel. Nesta mesma planilha, foi calculado a taxa de poluição de cada componente, no qual é o quociente da exergia de entrada dividido pelo trabalho produzido ou exergia do produto final do processo.

\section{RESULTADOS E DISCUSSÃO}

Conforme dito anteriormente, o fluxo de síntese de metanol foi refeito utilizando o mesmo software. A figura 3 demonstra uma simplificação do fluxograma da síntese do metanol, uma vez que a versão completa não caberá neste trabalho. Em cada seta, foi adicionado um medidor para a obtenção de valores do fluxo (temperatura, entalpia, entropia), para o cálculo das exergias correspondentes. 


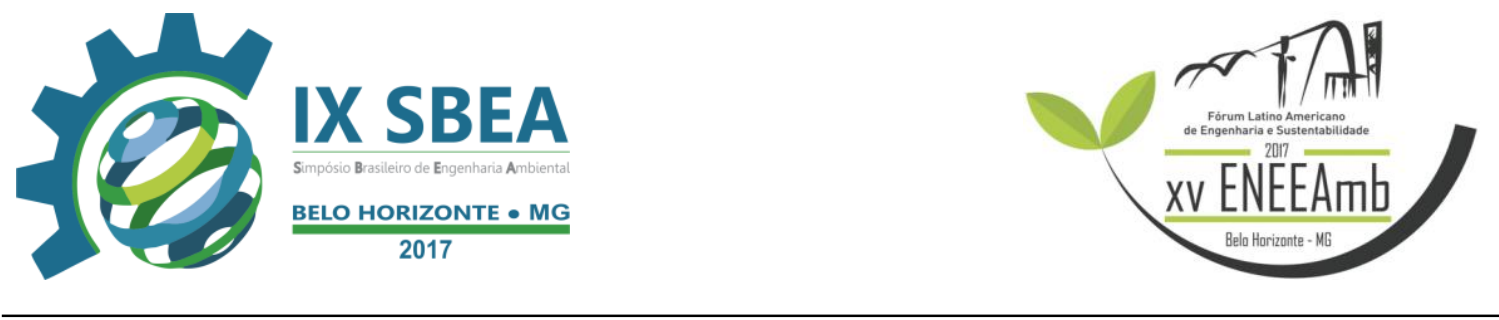

Figura 3 - Novo desenho da síntese de Metanol com o software COCO

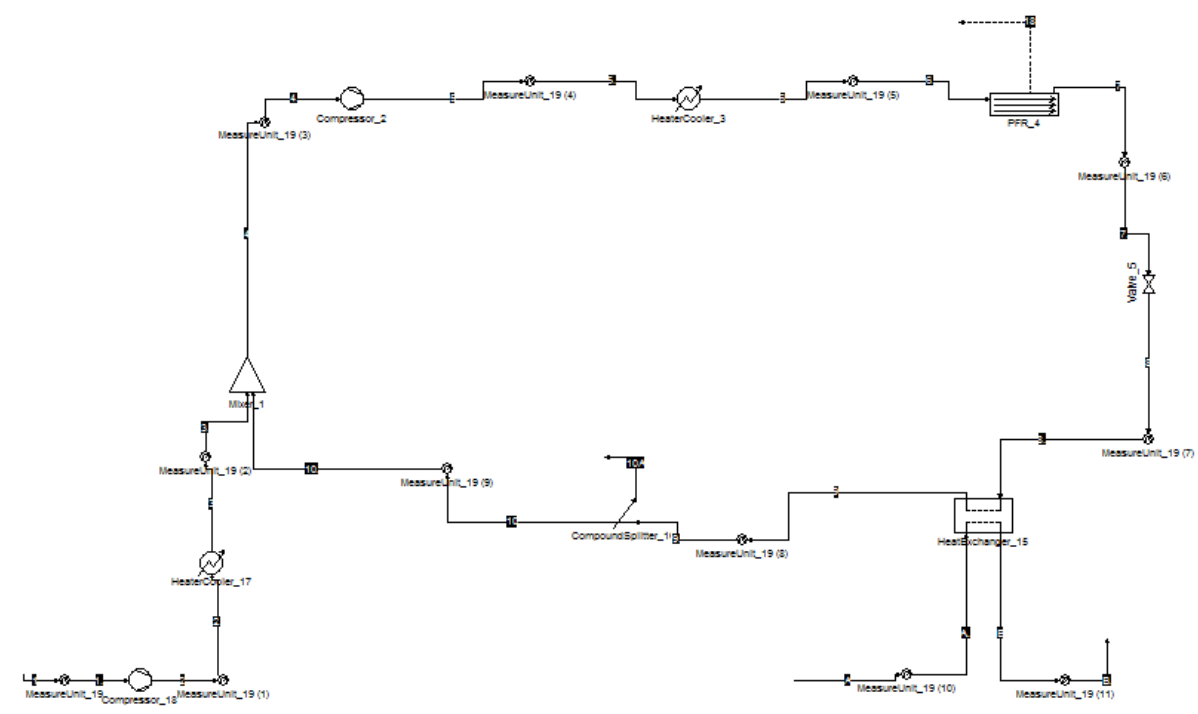

Fonte: Elaboração própria

As figuras 3, 4 e 5 a seguir demonstra alguns dos valores para o cálculo das exergias. A figura 3 ilustra as propriedades do fluido (pressão, temperatura, porcentagem de cada composto, vazão), enquanto a figura 4 ilustra as propriedades do fluido no estado gasoso e a figura 6 no estado líquido. É notável que após a entrada do fluido no reator, o componente existira tanto no estado líquido quanto no gasoso.

Figura 4 - Dados do fluido em cada componente (pressão, temperatura, fração de massa)

\begin{tabular}{|c|c|c|c|c|c|c|c|c|}
\hline name & para o compressor & para o trocador de calor & para o reator & para a válvula & para o trocador de calor tipo tubo & para o separador de gáses & para o misturador & unit \\
\hline \multicolumn{9}{|l|}{$\checkmark$ Overall } \\
\hline pressure & 2159.753 & 9167.9344 & 9167.9344 & 9168.0167 & 2169.753 & 2169.753 & 2169.753 & $\mathrm{kPa}$ \\
\hline temperature & 24.727253 & 178.43063 & 100 & 141.34264 & 108.20914 & 24.99951 & 24.99951 & ${ }^{\circ} \mathrm{C}$ \\
\hline mass fraction [Carbon monoxide] & 0.0056586534 & 0.0056586534 & $0.00565 \ldots$ & 0.0056 & 0.0056579142 & 0.0056586568 & 0.0075023187 & \\
\hline mass fraction [Carbon dioxide] & 0.93687255 & 0.93687255 & 0.93687255 & 0.7206 & 0.72069604 & 0.72081667 & 0.95566784 & \\
\hline mass fraction [Hydrogen] & 0.0574688 & 0.0574688 & 0.0574688 & 0.0277. & 0.027762381 & 0.027779071 & 0.036829842 & \\
\hline mass fraction [Water] & 0 & 0 & 0 & 0.0884 & 0.088490698 & 0.088441321 & 0 & \\
\hline mass fraction [Methanol] & 0 & 0 & 0 & 0.1573. & 0.15739297 & 0.15730428 & 0 & \\
\hline flow & 3. $3910344 \mathrm{e}-05$ & 3. $3910344 \mathrm{e}-05$ & $3.39103 \ldots$ & $3.3910 \ldots$ & 3. $3910321 \mathrm{e}-05$ & 3. $3910324 \mathrm{e}-05$ & $2.5577011 \mathrm{e}-05$ & $\mathrm{~kg} / \mathrm{s}$ \\
\hline MW & 20.000788 & 20.000788 & 20.000788 & 24.891785 & 24.891785 & 24.888354 & 24.843031 & $\mathrm{~kg} / \mathrm{kmo}$ \\
\hline
\end{tabular}

Fonte: $\mathrm{COCO}$ 


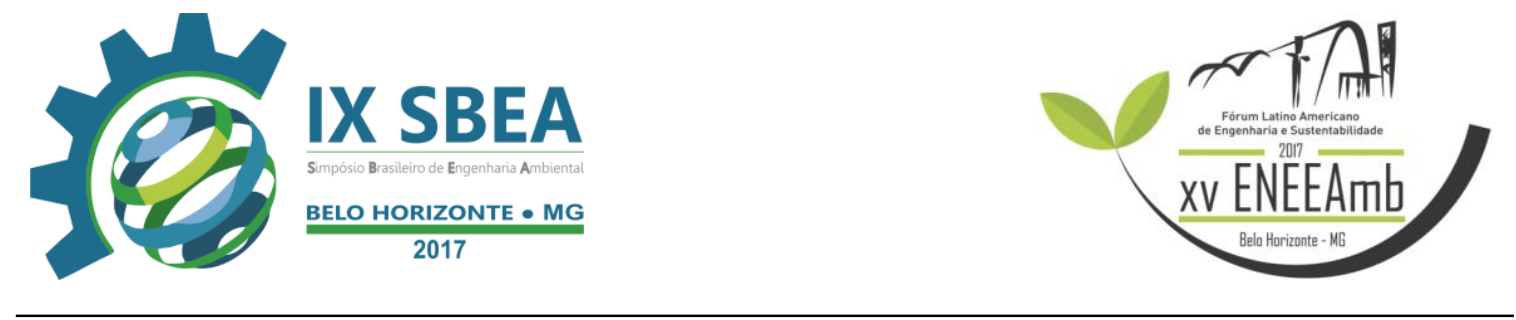

Figura 6 - Dados do fluido em cada componente no estado gasoso (entalpia, densidade, entropia, energia interna, número de Gibbs, calor específico)

\begin{tabular}{|c|c|c|c|c|c|c|c|c|}
\hline name & para o compressor & para o trocador de calor & para o reator & para a válvula & para o trocador de calor tipo tubo & para o separador de gáses & para o misturador & unit \\
\hline \multicolumn{9}{|c|}{ Vapor properties } \\
\hline density & 17.955871 & 49.250339 & 61.374095 & 72.923746 & 17.854715 & 22.631209 & 22.720271 & $\mathrm{~kg} / \mathrm{m}^{2}$ \\
\hline internalenergy & -133.51475 & 49.973211 & -59.587267 & -13.349137 & -20.22558 & -110.71175 & -110.01631 & $\mathrm{~kJ} / \mathrm{kg}$ \\
\hline enthalpy & -13.233587 & 236.12288 & 89.79065 & 112.37146 & 101.29711 & -14.837373 & -14.51776 & $\mathrm{~kJ} / \mathrm{kg}$ \\
\hline enthalpyF & -8412.5895 & -8163.2331 & -8309.5653 & -8374.7741 & -8370.587 & -8573.0312 & -8589.2067 & $\mathrm{~kJ} / \mathrm{kg}$ \\
\hline entropy & -1.0105253 & -0.92580439 & -1.2817394 & -0.75833352 & -0.32034489 & -0.80928985 & -0.81703638 & $\mathrm{~kJ} / \mathrm{kg} \mathrm{K}$ \\
\hline entopyF & 7.3004573 & 7.3851782 & 7.0292432 & 6.4997757 & 6.9400222 & 6.3053616 & 6.2613952 & $\mathrm{~kJ} / \mathrm{kgK}$ \\
\hline gibssEnergy & 287.7789 & 654.19821 & 568.07169 & 426.69512 & 223.46356 & 226.452 & 229.08124 & $\mathrm{~kJ} / \mathrm{kg}$ \\
\hline heatCapacityCp & 1.6836019 & 1.8685465 & 1.8686024 & 1.7605586 & 1.5334855 & 1.42806 & 1.4185431 & $\mathrm{~kJ} / \mathrm{kgK}$ \\
\hline
\end{tabular}

Fonte: $\mathrm{COCO}$

Figura 5 - Dados do fluido em cada componente no estado líquido (entalpia, densidade, entropia, energia interna, número de Gibbs, calor específico)

\begin{tabular}{|c|c|c|c|c|c|c|c|c|}
\hline name & para o compressor & para o trocador de calor & para o reator & para a válvula & para o trocador de calor tipo tubo & para o separador de gáses & para o misturador & unit \\
\hline \multicolumn{9}{|c|}{ Liquid properties } \\
\hline density & & & & 750.66764 & 807.45356 & 855.47276 & & $\mathrm{~kg} / \mathrm{m}^{2}$ \\
\hline internalenergy & & & & -1267.593 & -1565.5824 & -1652.9149 & & $\mathrm{~kJ} / \mathrm{kg}$ \\
\hline enthalpy & & & & -1255.3799 & -1562.8952 & -1650.3786 & & $\mathrm{~kJ} / \mathrm{kg}$ \\
\hline enthalpyf & & & & -10906.223 & -11692.25 & -10539.246 & & $\mathrm{~kJ} / \mathrm{kg}$ \\
\hline entropy & & & & -3.4001624 & -4.1216707 & -4.5123853 & & $\mathrm{~kJ} / \mathrm{kgk}$ \\
\hline entropyF & & & & 5.2510699 & 4.9220487 & 3.9120006 & & $\mathrm{~kJ} / \mathrm{kgk}$ \\
\hline gibbsEnergy & & & & 153.96238 & 8.9415466 & -305.01316 & & $\mathrm{~kJ} / \mathrm{kg}$ \\
\hline heatCapacityCp & & & & 4.348426 & 4.3260054 & 3.8401785 & & $\mathrm{~kJ} / \mathrm{kg} \mathrm{K}$ \\
\hline
\end{tabular}

Fonte: $\mathrm{COCO}$

Com todos estes valores, foi possível pelo software o cálculo das exergias de cada componente, para que os mesmos possam ser reescritos em uma tabela no Excel. Utilizando este aplicativo do pacote Office, a tabela 2 ilustra a taxa de destruição de cada componente. Vale ressaltar que para cada componente foram utilizados critérios diferentes para a obtenção do trabalho e da exergia de entrada. Para o misturador, a válvula e o separador de gáses, foi considerado que o trabalho é exergia de saída calculada no ponto correspondente (equação 1). Para o compressor, foi considerado o trabalho (W) uma vez que o valor existe neste componente. Para os trocadores de calor, foram considerados o calor trocado (Q). Finalmente, para o reator, a diferença entre as exergias dos produtos da reação mais a exergia pela equação 1 foi considerada a exergia de entrada, e o trabalho foi considerado como a exergia de saída. 


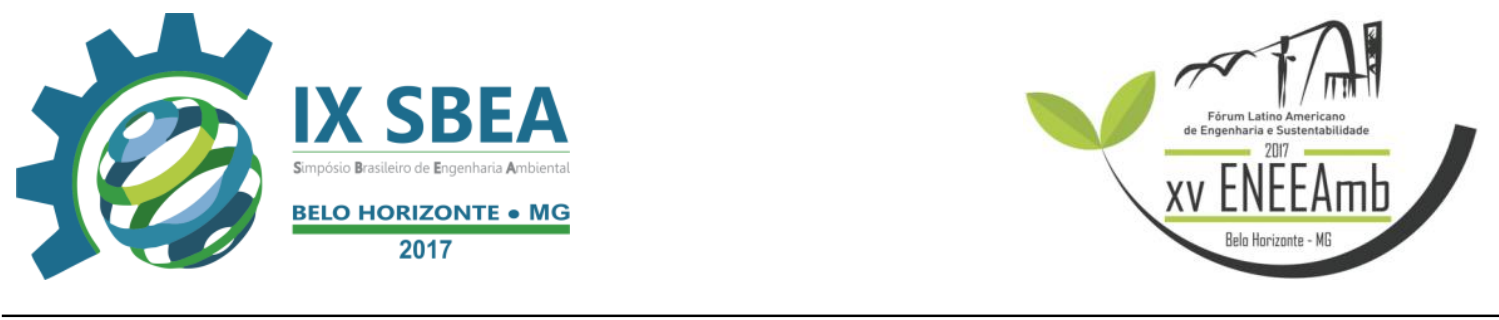

Tabela 2 - Taxa de destruição de cada componente do ciclo de síntese de metanol

\begin{tabular}{|c|c|c|c|}
\hline Componente & $\begin{array}{c}\text { Trabalho } \\
(\mathbf{W})\end{array}$ & $\begin{array}{c}\text { Exergia de entrada } \\
(\mathbf{W})\end{array}$ & $\begin{array}{c}\text { Taxa de } \\
\text { destruição }\end{array}$ \\
\hline Misturador & 5,50 & 10,1142 & 1,83969 \\
\hline Compressor & 10,11 & 6,7034 & 0,66277 \\
\hline Trocador de Calor & 16,00 & 0,0007 & 0,00004 \\
\hline Reator & 29,11 & 8,8669 & 0,30460 \\
\hline Válvula Borboleta & 8,87 & 4,8308 & 0,54481 \\
\hline $\begin{array}{c}\text { Trocador de Calor tipo } \\
\text { Tubo }\end{array}$ & 4,83 & 11,9152 & 2,46649 \\
\hline Separador de vapor & 3,15 & 5,8561 & 1,85856 \\
\hline
\end{tabular}

Fonte: Elaboração própria

Segundo Bejarano (2004), quando a taxa de poluição total é maior que 1, as emissões dos processos provocam um grande impacto no meio ambiente, mas se estiver entre 0 e 1, os processos apresentam um impacto ambiental em função de limitações tecnológicas dos processos de conversão de energia.

Desta forma, considerando o sistema como um todo, ele apenas tem um trocador de calor, o separador e o misturador como grandes impactantes ao meio ambiente. Já os demais componentes não apresentam um impacto significativo. Esses valores pequenos na realidade já eram esperados pelo fato da síntese de metanol ser um processo alternativo para outras fontes de energia, já que o metanol não polui o meio ambiente como outros gases convencionais.

\section{CONCLUSÕES/RECOMENDAÇÕES}

O software COCO provou ser uma ferramenta não só para a análise termodinâmica de ciclos convencionais como o Rankine, mas também como uma ferramenta de análise ambiental. Isto pelo fato do software possibilitar cálculos como o trabalho e as exergias em cada componente de vários ciclos, tais como o da síntese de metanol, conforme foi apresentado no trabalho.

Os resultados obtidos foram bons, e demonstram que a produção de metanol pode ser um passo à frente para a produção de combustíveis cujo impacto ambiental é o menor possível. Da mesma forma que o COCO pode ser uma ferramenta importante para estudos de outros combustíveis. 


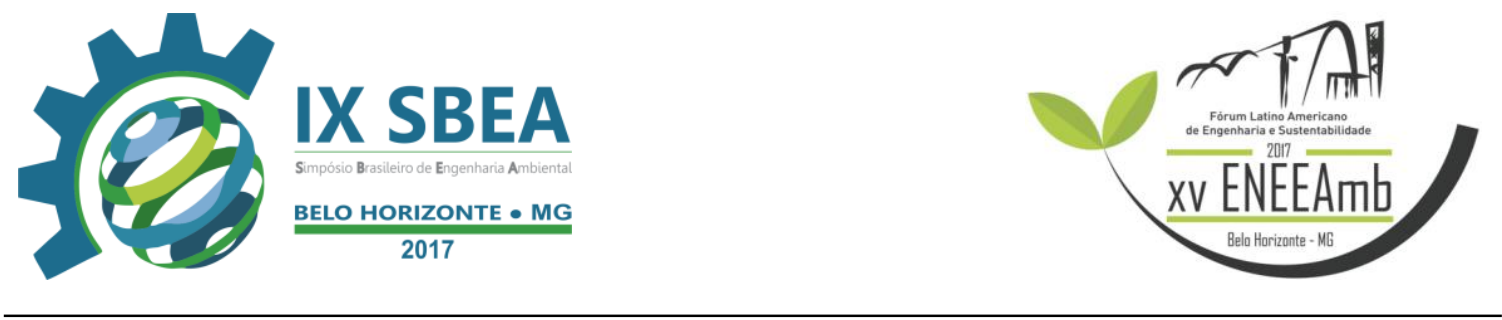

\section{REFERÊNCIAS BIBLIOGRÁFICAS}

\section{Livro}

MORAN, Michael J., SHAPIRO, Howard N. Fundamentals of Engineering Thermodynamics. 5 th Edition 2004.

\section{Artigo de periódico}

ACKER, Thomas L., MORGAN, Eric R. Practical Experience with a Mobile Methanol

Synthesis Device. Northern Arizona University Department of Mechanical Engineering.

\section{Monografia, dissertação e tese}

ROJAS, Sílvia Palma. ANÁLISE EXERGÉTICA, TERMOECONÔMICA E AMBIENTAL DE UM SISTEMA DE GERAÇÃO DE ENERGIA. ESTUDO DE CASO: USINA TERMOELÉTRICA UTE - RIO MADEIRA. Brasília, 195 p., 2007. Dissertação (mestrado) - Universidade de Brasília. Disponível em: http://repositorio.unb.br/bitstream/10482/2826/1/2007_SilviaIlenaPRojas.pdf

Trabalho em evento

PINTO E SILVA, Rafael. COCO 2.07 Users Guide Summary. Northern Arizona University Sustainable Energy Solutions.

\section{Internet}

VAN BATEN, Jasper. COCO Software. Disponível em: http://www.cocosimulator.org/index_download.html. 